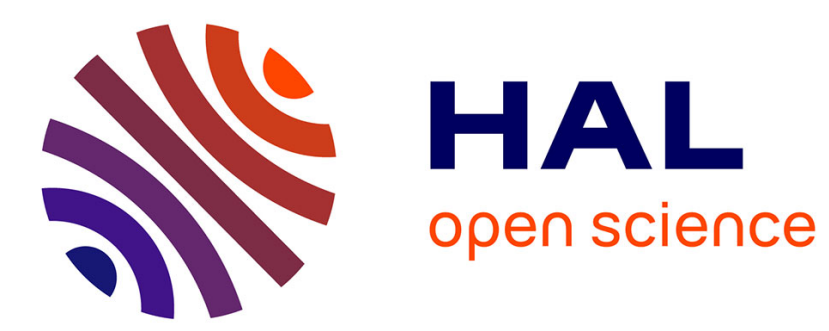

\title{
Seismic influence on the VLF transmitter signal intensity measured by the low-altitude satellite DEMETER
}

\author{
F. Němec, M. Liška, Michel Parrot
}

\section{To cite this version:}

F. Němec, M. Liška, Michel Parrot. Seismic influence on the VLF transmitter signal intensity measured by the low-altitude satellite DEMETER. The European Physical Journal. Special Topics, 2021, 230 (1), pp.227-245. 10.1140/epjst/e2020-000271-1 . insu-03157259

\section{HAL Id: insu-03157259 \\ https://hal-insu.archives-ouvertes.fr/insu-03157259}

Submitted on 23 Apr 2021

HAL is a multi-disciplinary open access archive for the deposit and dissemination of scientific research documents, whether they are published or not. The documents may come from teaching and research institutions in France or abroad, or from public or private research centers.
L'archive ouverte pluridisciplinaire HAL, est destinée au dépôt et à la diffusion de documents scientifiques de niveau recherche, publiés ou non, émanant des établissements d'enseignement et de recherche français ou étrangers, des laboratoires publics ou privés. 


\title{
Seismic influence on the VLF transmitter signal intensity measured by the low-altitude satellite DEMETER
}

\author{
F. Němec ${ }^{1, a}$, M. Liška ${ }^{1}$, and M. Parrot ${ }^{2}$ \\ ${ }^{1}$ Faculty of Mathematics and Physics, Charles University, 12116 Prague, Czech Republic \\ ${ }^{2}$ LPC2E/CNRS, UMR7328 Orléans, France
}

Received 7 October 2020 / Accepted 7 October 2020

Published online 19 January 2021

\begin{abstract}
We present results of a statistical study of a possible influence of the seismic activity on the intensity of very low frequency (VLF) transmitter signals observed by a low-altitude satellite. Electric field measurements performed by the Detection of Electro-Magnetic Emissions Transmitted from Earthquake Regions (DEMETER) satellite during its entire mission spanning almost 6.5 years were used. Among various VLF transmitter signals detected, we focused particularly on the NWC and JJI transmitters, because of their favorable locations close to seismically active areas. We evaluate the intensities of the detected transmitter signals at the times when they passed in the vicinity of an imminent earthquake during the propagation in the Earth-ionosphere waveguide, and we statistically compare them with the intensities measured at the times when there was no earthquake present. Only earthquakes with magnitudes larger than or equal to 5 and depths shallower than or equal to $40 \mathrm{~km}$ were considered in the analysis. Moreover, due to the low intensity of detected transmitter signals during the day, the analysis is limited exclusively to the nightside. Although the amount of relevant data is rather low, the obtained results show that there is a decrease of the detected intensity shortly (0-3 hours) after the times of the main shocks observed both for the NWC and JJI transmitter signals. The effect is spatially rather limited, observed when the signal passes within about 4 degrees from the earthquake epicenter. The intensity decrease appears to be consistent with acoustic-gravity waves propagating from the earthquake region and influencing the bottom of the ionosphere.
\end{abstract}

\section{Introduction}

A considerable number of electromagnetic effects in the ionosphere possibly related to the seismic activity have been reported over the last few decades [1-3]. Related theoretical development suggests that these are due to litosphere-atmosphere-ionosphere coupling [4-8]. Given that they may occur not only after the earthquake occurrence, but also shortly before the time of the main shock, they might - if confirmed and

a e-mail: frantisek.nemec@gmail.com 
understood - eventually serve as short-time precursors of an imminent earthquake $[9,10]$.

Possible seismic-related electromagnetic effects may occur in various frequency ranges from ultra low frequency (ULF, from $1 \mathrm{mHz}$ to $3 \mathrm{~Hz}$ ) through extra low frequency (ELF, from $3 \mathrm{~Hz}$ to $3 \mathrm{kHz}$ ) up to very low frequency (VLF, from $3 \mathrm{kHz}$ to $30 \mathrm{kHz}$ ), and on various time scales, from several minutes up to a few months before an earthquake [11-16]. However, there is still not an overall agreement on the nature of the observed effects, as both intensity increases $[9,14,17,18]$ and wave intensity attenuations [19-23] were reported. In fact, the very existence of this exciting phenomenon is still doubtful [24-28], as - if existing - the seismic related effects are very weak and they can be easily hidden in common natural variations of electromagnetic waves.

This complication can be effectively overcome by large statistical studies including many different earthquakes and using a variation of a superposed epoch analysis. A systematic analysis of ELF/VLF electromagnetic wave intensity measured by the low-altitude DEMETER satellite revealed that there might be a statistically significant decrease of the wave intensity at frequencies of about $1.7 \mathrm{kHz}$ observed shortly (0-4 hours) before the times of earthquakes during the night [20]. This result was confirmed using a larger data set [21], and eventually re-confirmed using the entirety of the DEMETER data measured during the mission duration $[22,23]$. It was suggested that the observed effect might be due to changes in the Earth-ionosphere waveguide $[29,30]$ and a related increase of the waveguide critical frequency [31]. This seems to be consistent with subionospheric VLF signal perturbations possibly related to earthquakes $[1,32]$ and to ion density variations reported in low-altitude satellite data [33-37]. A decrease of the VLF transmitter intensity observed by the DEMETER spacecraft was also reported in the first 2 years of data, and it was speculated that it might be due to the influence of atmospheric gravity waves induced by the gas-water release from the earthquake preparatory zone [19]. Case studies related to powerful earthquakes were further reported [38-45]. These studies typically reported a decrease of detecting the transmitter signal intensity, which might occur as long as several days before the time of the main shock [46,47]. However, occasional signal enhancements were also reported [48].

We investigate possible seismic-related variations of the intensity of VLF transmitter signals observed by the DEMETER spacecraft using the data measured during the entire duration of the mission (about 6.5 years). A variation of the two-step data processing method, which allows us to account for non-seismic related signal variations, is applied [20]. The used data sets are introduced in Section 2. The way of associating the recorded signals with individual earthquakes and subsequent statistical analyses is described in Sections 3 and 4, respectively. Section 5 contains the results of the statistical analysis of the seismic influence on the transmitter signal intensity. These are discussed in Section 6, and they are briefly summarized in Section 7.

\section{Data sets}

DEMETER was a low-altitude satellite operating at a circular polar orbit with an altitude of about $700 \mathrm{~km}$ (the original altitude of about $710 \mathrm{~km}$ was decreased to about $660 \mathrm{~km}$ in December 2005). It was active since June 2004 until December 2010. The orbit of the satellite was almost Sun-synchronous; the north-to-south half-orbits corresponded to the local time of about 10:30 hours, while south-to-north half-orbits corresponded to the local time of approximately 22:30 hours. This allows us to conveniently separate the nighttime and the daytime data. DEMETER measurements took place at geomagnetic latitudes between $-65^{\circ}$ and $65^{\circ}$. Both electric and magnetic 
(a)

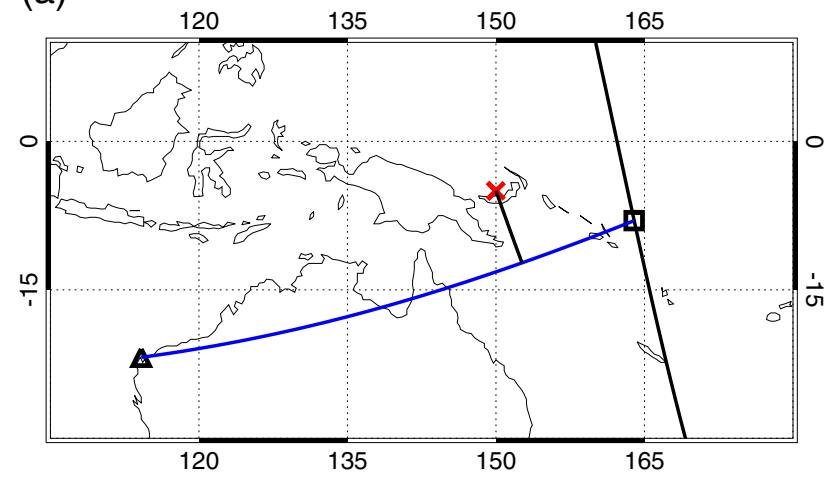

(b)

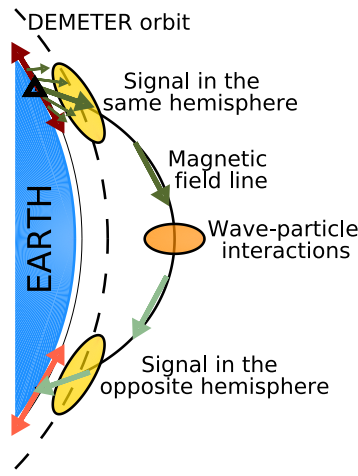

Fig. 1. (a) A scheme of the situation used to evaluate the earthquake distance. The location of a transmitter is shown by the black triangle. The location of the earthquake epicenter is shown by the red cross. The black curve shows the projection of the DEMETER orbit. The great circle path between the transmitter location and the spacecraft location at a given time is shown by the blue curve. The distance of the particular measurement from the earthquake considered for the performed analysis is then defined as the shortest distance between the earthquake epicenter and the great circle path. (b) A sketch of the transmitter signal propagation along the magnetic field line to the conjugate hemisphere. The transmitter location is shown by the black triangle at the top. Its signal propagates in the Earth-ionosphere waveguide (dark red arrows) and eventually leaks through the ionosphere to higher altitudes (dark green arrows) where it is measured by the DEMETER spacecraft. It further propagates roughly along the magnetic field line to the opposite hemisphere. The signal observed in the conjugate hemisphere may be significantly modified due to wave-particle interactions taking place in the equatorial region.

field measurements were performed. However, VLF magnetic field data suffer from a significant amount of onboard interferences, and only the electric field data are thus used in the present paper. In a continuously active survey mode, the VLF electric field data cover the frequency range from $20 \mathrm{~Hz}$ to $20 \mathrm{kHz}$ with a frequency resolution of about $19.5 \mathrm{~Hz}$ and a time resolution of about $2 \mathrm{~s}$. More detailed description of the electric field instrument was given in a dedicated instrument paper [49]. We use data from the entire time period when the DEMETER satellite was active (approximately 6.5 years). The Global Centroid-Moment-Tensor catalog (http://www.ldeo. columbia.edu/ gcmt/projects/CMT/catalog/jan76_dec13.ndk) was used as a source of the seismic activity data, providing us with the time, magnitude, location, and depth of hypocenter of earthquakes which occurred all over the world during the time period of interest.

\section{Data processing}

In order to evaluate a possible seismic influence on the transmitter signal intensity, we suggest a scheme shown in Figure 1a. The geographic location of a considered transmitter is depicted by the black triangle in the lower left part of the figure. The black curve in the right-hand side of the figure corresponds to the projection of the DEMETER spacecraft orbit. The spacecraft location at a selected time is then depicted by the black square. The blue curve shows the great circle path between the transmitter and the spacecraft location, corresponding to the path of the transmitter signal propagation in the Earth-ionosphere waveguide. Assuming that an earthquake 


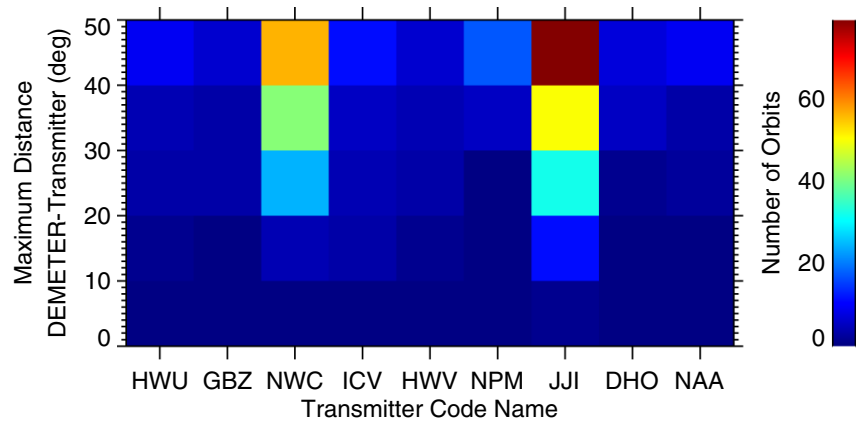

Fig. 2. Number of DEMETER half-orbits with data potentially influenced by seismic effects, assuming that these effects occur exclusively during the night, last for 1 hour around the time of the main shock, and they affect the transmitter signal propagation at great circle paths within 3 degrees. The number of relevant half-orbits is color coded according to the scale on the right-hand side as a function of the considered transmitter (abscissa) and maximum allowed distance between DEMETER and the transmitter (ordinate).

occurs close to this path, it may potentially influence the waveguide properties and thus also the wave intensity detected by the spacecraft. The position of a hypothetical earthquake is marked by the red cross. The short black curve connecting the earthquake location and the great circle path represents the shortest distance between the earthquake epicenter and the signal propagation path, which is considered when evaluating whether a given earthquake is close enough to have a detectable effect or not. We note that another possibility of the transmitter signal propagation would be the propagation nearly along the magnetic field lines to the opposite hemisphere. A sketch of the situation is shown in Figure 1b. It shows a part of the Earth, a transmitter (black triangle at the top), and a corresponding magnetic field line. The dashed circular curve corresponds to the DEMETER orbit. A VLF signal generated by the transmitter propagates in the Earth-ionosphere waveguide, as shown by the dark red arrows at the top. The dark green arrows depict an eventual leakage of the transmitter signal through the ionosphere to higher altitudes. There it is detected by DEMETER, as shown by the yellowish ellipse. The signal, however, further propagates, roughly along the magnetic field line, to the opposite hemisphere (light green arrows). There, close to the magnetically conjugate point, it again reaches the DEMETER altitudes and can be detected by the spacecraft. Finally, the signal may make it down to the Earth-ionosphere waveguide in the conjugate hemisphere and propagate therein (light red arrows). Importantly, as the waves propagate through the equatorial region, their intensity and spectral features can be significantly altered due to wave-particle interactions $[50,51]$. This vastly complicates a proper characterization of wave intensities in the conjugate region and limits their usability for the analysis of possible seismic-related effects. Only DEMETER satellite measurements performed at the geomagnetic hemisphere of the transmitter are thus used in the present study.

Possible variations of the VLF transmitter signal intensity related to the seismic activity are expected (if existent) to be very weak. In order to distinguish them from other intensity variations of a comparable amplitude, a statistical analysis including many different events is needed. It is thus desirable to identify VLF transmitters whose signal would often encounter an imminent earthquake on its great circle path to DEMETER. The results of this identification of the transmitters most favorable for the intended statistical analysis are shown in Figure 2. We considered the 12 continuously operating VLF transmitters from Table 1 [52], which are readily detectable by the DEMETER spacecraft. Out of these, we disregard the 3 Russian Alpha 
transmitters, which alternate between three different frequencies, and are thus not optimally suited for our purpose. This leaves us with 9 transmitters possibly suitable for the analysis. In order to estimate the total number of individual earthquakes ("events") possibly influencing the transmitter signal path over the entire DEMETER mission duration, we assume that: (i) only earthquakes with magnitudes larger than or equal to 5.0 and depths shallower than or equal to $40 \mathrm{~km}$ can influence the transmitter intensity, (ii) a given earthquake affects the transmitter signal propagation at great circle paths within 3 degrees from the earthquake epicenter, (iii) the effect lasts for 1 hour around the time of the main shock, and (iv) the effect occurs only during the local night. Although these assumptions are clearly somewhat arbitrary, they provide us for each transmitter with a rough estimate of the number of relevant events. Most importantly, the obtained results are rather independent of the exact assumptions made, as they remain qualitatively the same.

Figure 2 shows the color coded number of events (i.e., number of DEMETER orbits for which the transmitter-DEMETER great circle path was potentially affected by an earthquake) as a function of the transmitter code name (abscissa) and maximum allowed distance between the transmitter and DEMETER measurements at a given time (ordinate). It can be seen that the number of events is generally rather low, with two transmitters standing out clearly. The first of them is the NWC transmitter (latitude $-21.82^{\circ}$, longitude $114.17^{\circ}$, operating frequency $19.8 \mathrm{kHz}$ ), which is the most powerful VLF transmitter operating in the southern hemisphere. The second transmitter resulting in considerable number of events is the JJI transmitter (latitude $32.04^{\circ}$, longitude $130.81^{\circ}$, operating frequency $22.2 \mathrm{kHz}$ ), which has a lower output power, but it is favorably located close to seismically active regions. The maximum considered distance between the transmitter location and the location of the DEMETER spacecraft clearly strongly affects the results. This parameter expresses the situation that the spatial extent of the transmitter signal is limited, i.e., only satellite measurements not too far from the satellite location should be considered. We express this distance in the units of angular degrees, i.e., one degree corresponds to about $110 \mathrm{~km}$. The actual value of this parameter is clearly related to the transmitter power output, penetration characteristics of the ionosphere, and transmitter signal attenuation in the Earth-ionosphere waveguide.

Although the statistical analysis presented in Section 4 would in principle allow us to combine signal intensity variations from all relevant transmitters together, we opted not to do so. Considering that these transmitters operate at different frequencies (and locations), the natural background variations for each of them would be slightly different, as might be also underlying physical mechanisms responsible for possible observed effects. Taking into account that the number of suitable events for other than the NWC and JJI transmitters is very low, using only the NWC and JJI transmitter data results in disregarding only small amount of data and provides us with a better picture of the situation. Additionally, analyzing the signals of these transmitters separately allows us for an independent check whether the same intensity variations are observed for the two considered transmitters. Only the NWC and JJI transmitter signals are thus used in the analysis.

The geographic locations of the NWC and JJI transmitters are shown in Figure 3 by the black triangle and black diamond, respectively. The black curve shows the geomagnetic equator. The NWC transmitter signal intensity is thus considered as potentially affected by the seismic activity only when measured at the times when DEMETER is located in the southern hemisphere, and the JJI transmitter signal intensity is considered as potentially affected by the seismic activity only when measured at the times when DEMETER is located in the northern hemisphere. Otherwise, the transmitter signal would propagate between the hemispheres primarily along the magnetic field lines, being influenced by wave-particle interactions in the 

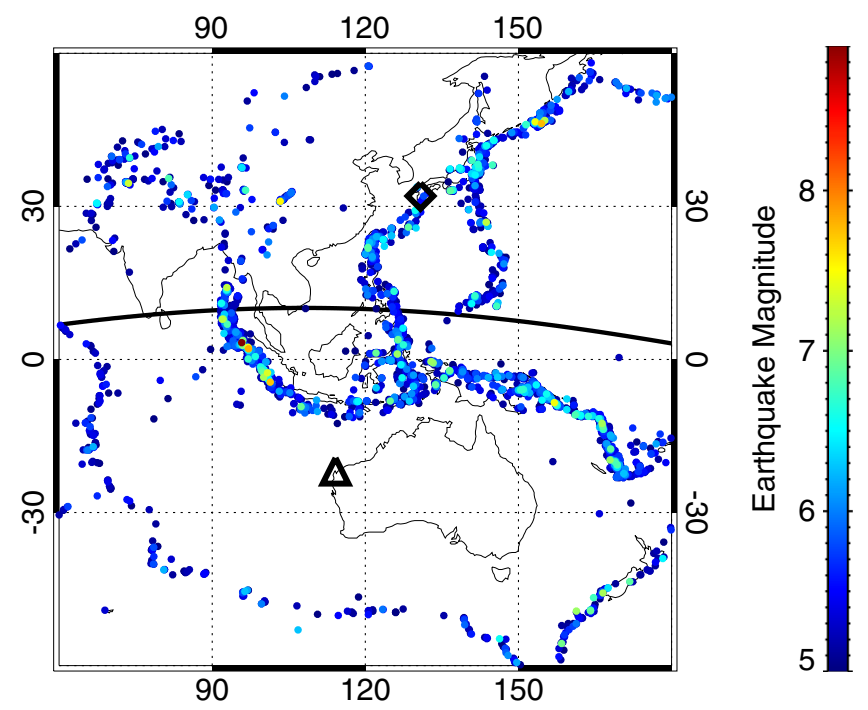

Fig. 3. Geographic locations of earthquakes with magnitudes larger than or equal to 5 and depths less than or equal to $40 \mathrm{~km}$ that occurred in the region of interest during the duration of the DEMETER mission. The magnitude of individual earthquakes is color coded according to the color scale on the right-hand side. The black curve shows the geomagnetic equator. The geographic locations of the NWC and JJI transmitters are shown by the black triangle and black diamond, respectively.

equatorial region, and a possible seismic influence on the great circle path could thus not be properly evaluated. The color points correspond to the locations of individual earthquakes with magnitudes larger than or equal to 5.0 and depths shallower than $40 \mathrm{~km}$ that occurred during the DEMETER mission duration, with the magnitude color coded according to the color scale on the right-hand side. It can be seen that both the NWC and JJI transmitters occur in the vicinity of seismically active regions and, consistently with the results from Figure 2, are thus optimally suited for the intended analysis.

\section{Statistical analyses}

Geographic maps of NWC and JJI transmitter signal intensities detected by the DEMETER spacecraft are depicted in Figures 4 and 5, respectively. Color coded are median power spectral densities in individual $2^{\circ} \times 2^{\circ}$ latitude-longitude bins. Seven frequency bins of the DEMETER ICE instrument (19.53 Hz each) centered at the $19.8 \mathrm{kHz}$ frequency of the NWC transmitter were used for the analysis in Figure 4. The position of the NWC transmitter is marked by the black triangles. The frequency bins used when constructing Figure 5 were selected around the frequency of $17.8 \mathrm{kHz}$, which is the aliased frequency of the JJI transmitter, considering the sampling frequency of the DEMETER VLF measurements $40 \mathrm{kHz}$. The position of the JJI transmitter is marked by the black diamonds. The black curves correspond to the geomagnetic equator. Figures $4 \mathrm{a}$ and $5 \mathrm{a}$ correspond to the daytime intensity measurements, while Figures $4 \mathrm{~b}$ and $5 \mathrm{~b}$ correspond to the nightside intensity measurements. It can be seen that the transmitter signal intensity depends significantly on the local time of the observation. During the day, the ionization in the ionosphere is much larger than during the night, and the resulting signal attenuation during the 
(a)

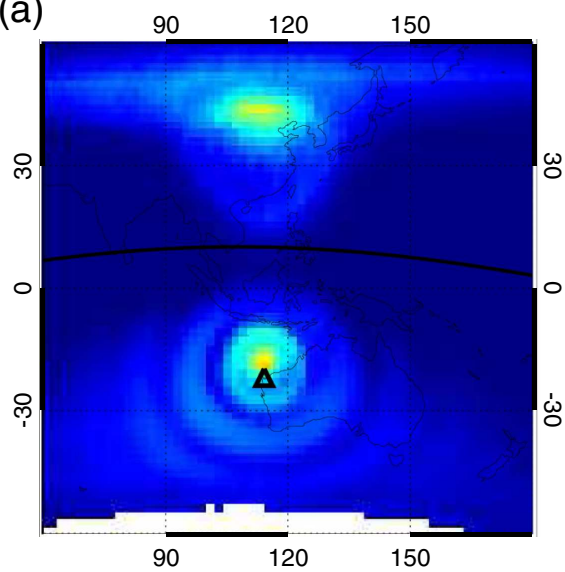

(b)

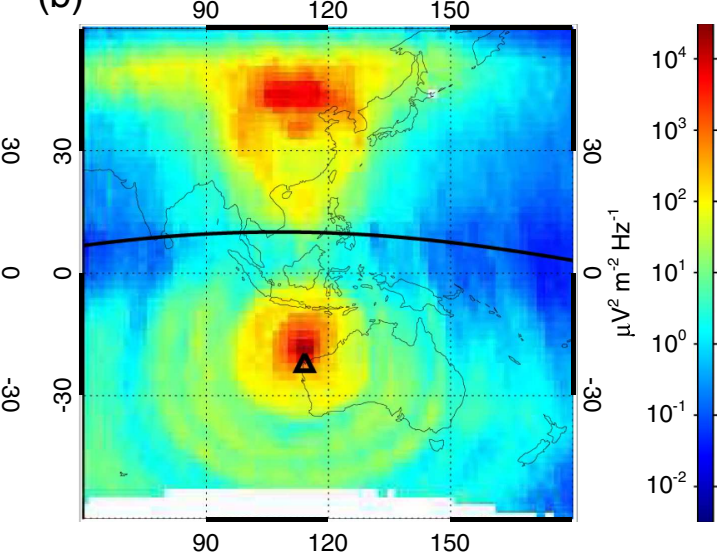

Fig. 4. Median power spectral density of electric field fluctuations measured by DEMETER at frequencies around $19.8 \mathrm{kHz}$, corresponding to the NWC transmitter frequency. (a) Data measured during the daytime half-orbits. (b) Data measured during the nighttime halforbits. The spatial resolution is $2^{\circ}$ both in longitude and latitude. The black triangle marks the location of the NWC transmitter. The black curve corresponds to the geomagnetic equator.

(a)

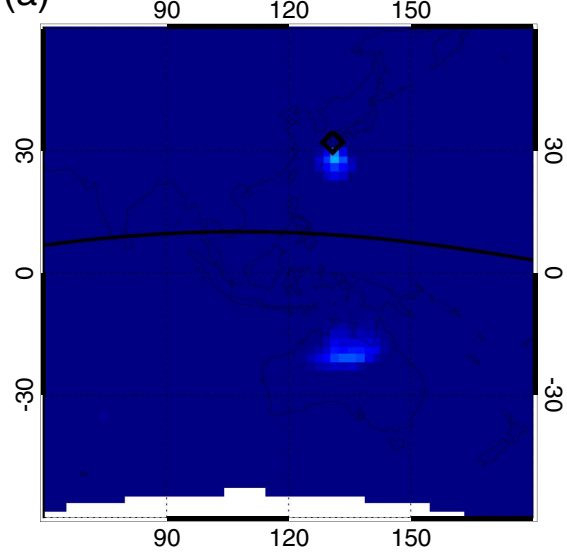

(b)

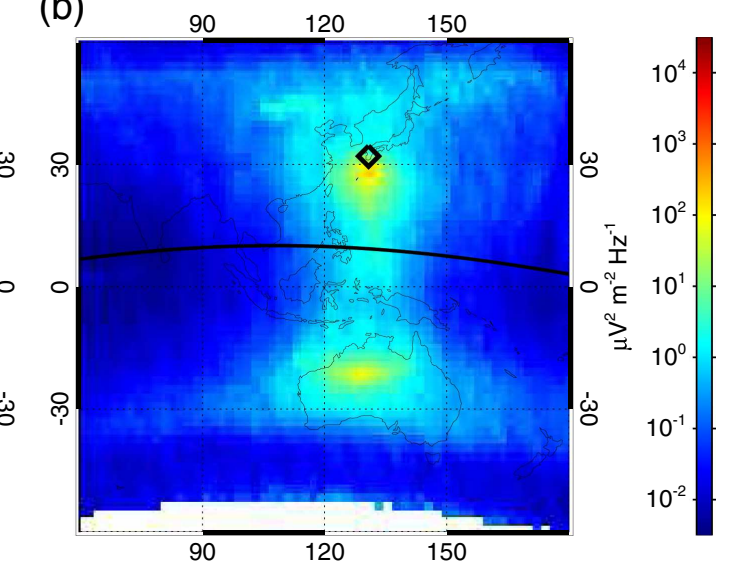

Fig. 5. Same as Figure 4, but for the JJI transmitter operating at frequency $22.2 \mathrm{kHz}$. The aliasing effect and DEMETER measurements performed at frequencies around $17.8 \mathrm{kHz}$ were used.

penetration through the ionosphere is thus roughly by an order of magnitude higher [54-56]. Additionally, the attenuation of a signal propagating in the Earth-ionosphere waveguide is generally larger during the day than during the night [57]. Consequently, the transmitter signal intensity detected by DEMETER is significantly larger during the night than during the day. Further, the transmitter signal intensity is - as expected - largest close to the transmitter locations and geomagnetically conjugated points. The slight shift of the peak intensity from the transmitter location toward the geomagnetic equator is due to the wave propagation between transmitter and the satellite in the direction along the ambient magnetic field rather than vertically [52]. We note that although the DEMETER altitude slightly changed over the mission duration, the signal attenuation at these high altitudes is extremely low, and 
this minor altitudinal change thus has principally no effect on the detected transmitter signal intensity [53]. Consequently, the full DEMETER data set is used in the presented analysis.

We further note that the time periods when the respective transmitters were not operating were determined by investigating the wave intensity close to the transmitter location [52] and excluded from the analysis. Finally, as discussed above, it is necessary to limit the analysis only to latitude-longitude intervals with a reasonable intensity of the transmitter signals. Specifically, we require the median intensity in a given latitude-longitude bin to be larger than $1 \mu \mathrm{V}^{2} \mathrm{~m}^{-2} \mathrm{~Hz}^{-1}$. If it is not, then all the measurements performed in a given spatial bin are excluded from the analysis. Although this intensity threshold is set slightly arbitrary, it roughly corresponds to the intensity safely larger than the background noise (see Figs. 4 and 5), but low enough in order not to exclude too much data. In any case, applying this intensity threshold removes the vast majority of the daytime data from the analysis, in particular in the case of the JJI transmitter. However, for both transmitters, the amount of intense-enough daytime data is very limited and effectively prevents us from performing the analysis. The subsequent analysis thus uses exclusively the nighttime data.

The detected transmitter signal intensity varies due to many different factors. In order to identify any possible signal intensity variations related to the earthquake occurrence, we would optimally need to account for these in some way. We thus apply a two-step data processing method based on the data processing formerly developed for natural VLF emissions [20]. The basic idea of the method is that, in the first step, we construct a long-term "map" of the wave intensity parameterized by relevant controlling factors, which is used to express "how intense a signal is expected to be at a given location under given conditions". Then, in the second step of the data processing, we evaluate the wave intensities measured in the vicinity of earthquakes by comparing them with the intensities expected according to the first step of the data processing. The actual application of the method to the specific analyzed situation of the NWC and JJI VLF transmitters is described below.

When constructing the long-term map of the transmitter signal intensity, the following parameters are considered as the most relevant controlling factors: (i) geographic longitude of the spacecraft $\left(2^{\circ}\right.$ resolution, i.e., 180 bins), (ii) geographic latitude of the spacecraft $\left(2^{\circ}\right.$ resolution, i.e., 90 bins), (iii) local time (daytime vs nighttime, i.e., 2 bins), and iv) season of the year (January-March, April-June, JulySeptember, October-December, i.e., 4 bins). We have also investigated a possible influence of the geomagnetic activity represented by the Kp index, but principally no relation between the transmitter signal intensity and the geomagnetic activity was found (not shown, consistent with the statistical results [52]). The level of the geomagnetic activity is thus further not considered in the presented analysis. In order to characterize the transmitter signal intensities measured by DEMETER at given locations under given conditions, a histogram of the transmitter signal intensities measured for each combination of the four parameters was stored with a $0.1 \log \left(\mu \mathrm{V}^{2} \mathrm{~m}^{-2} \mathrm{~Hz}^{-1}\right)$ resolution.

A crucial point of this first step of the performed processing is that it allows us to evaluate whether the intensity measured at a given place under given conditions (i.e., day/night and season) is lower or larger than the long-term average and by how much. This can be conveniently expressed by a corresponding cumulative probability, i.e., a ratio of the measurements which took place at the same place and under the same conditions which are less intense than the actually measured value [20]. The values of the cumulative probability equal to 0 and 1 correspond to the lowest and largest intensities ever measured at a given location under given conditions, respectively. The value of the cumulative probability equal to 0.5 corresponds to a 
situation of the median intensity, when exactly one half of the intensities measured at a given place and under given conditions was less intense and one half more intense. Importantly, independently of the original intensity distribution, the values of the cumulative probability are uniformly distributed [20]. This allows us not only to compare the measured intensities with the expected long-term distribution, but also to conveniently evaluate the associated statistics.

In the second step of the data processing, we use the map of the transmitter signal intensities constructed in the first step and the associated cumulative probabilities to investigate whether there is a variation of the signal intensity related to the earthquake occurrence or not. The crucial point in this analysis is to determine whether the transmitter signal is located "close to an earthquake" or not. As discussed above, we consider a picture of an imminent earthquake possibly influencing the electron ionization/temperature at the bottom of the ionosphere, modifying thus inherently the Earth-ionosphere waveguide. The transmitter signal propagating therein would be modified, and its intensity change as compared to normal conditions might be detected. In order to possibly influence the transmitter signal intensity, the earthquake epicenter thus has to be close to the transmitter-DEMETER great circle path. This simple picture assumes the transmitter signal to propagate principally all the way to the spacecraft in the Earth-ionosphere waveguide, i.e., we disregard the signals which were ducted along the ambient magnetic field between the hemispheres. Consequently, only DEMETER measurements performed in the same hemisphere as the transmitter are considered for the evaluation of possible seismic-related effects.

In order to evaluate signal intensity variations possibly related to the seismic activity, we considered the time interval from 72 hours before the main shock times until 24 hours after the main shock times. Although seismic related effects might possibly occur more than three days before the main shock, one should consider that the sequence of processes on these longer time scales is surely slightly different for each earthquake. The effect would then occur at a slightly different time for each event, and the superposed epoch analysis would average out the effect. Also, should the considered time window be too long, too much data would be eliminated from the analysis in an attempt not to mix pre- and post-seismic effects (see below). Otherwise, the particular choice of the considered time window does not affect the obtained results. Only earthquakes with magnitudes larger than or equal to 5.0 and with depths of hypocenters shallower than or equal to $40 \mathrm{~km}$ were included in the analysis. This choice follows former results [20], and it is consistent with the intuitive expectations that no effect is to be observed for weak/very deep earthquakes. We note that any other (even non-seismic events) possibly affecting the detected transmitter signal intensity, which are not included in the analysis, would be randomly distributed with respect to the considered time intervals, i.e., they only introduce a random "noise" in the performed analysis.

DEMETER data for which an earthquake epicenter is within 10 degrees from the great circle path connecting the transmitter and the DEMETER location are then evaluated. If - for a given spacecraft position - more than one earthquake was close to the transmitter-DEMETER great circle path within the considered 96 hours long time window, we discard the data from the analysis. This is done in order not to mix pre- and post-seismic effects, and, moreover, to prevent a single piece of data to be included in the analysis several times [20]. The relevant data are then organized as a function of the distance between the earthquake epicenter and the transmitterDEMETER great circle path and the time to/from an earthquake. We use 1 hour time resolution (i.e., 96 time bins) and 1 degree resolution in distance (i.e., 10 distance bins). We then define a "probabilistic intensity" in each $96 \times 10$ bins as an average value of the respective cumulative probabilities minus 0.5. All relevant DEMETER data are used in the calculation. Finally, we normalize the probabilistic intensities by 
(a)

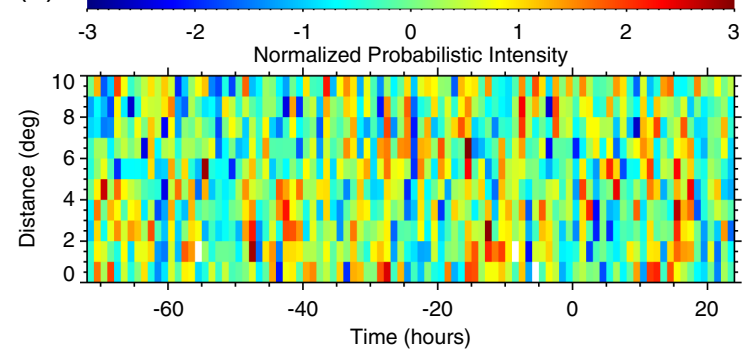

(b)

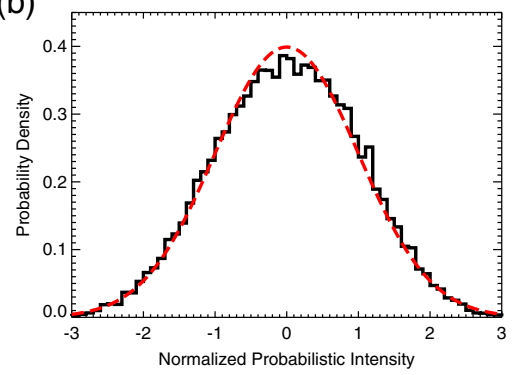

Fig. 6. (a) Normalized probabilistic intensities obtained for the NWC transmitter and a surrogate earthquake catalog are color coded as a function of the time relative to the times of the main shocks (abscissa) and distance of the NWC transmitter-DEMETER great circle paths from earthquake epicenters (ordinate). (b) Distribution of values of normalized probabilistic intensity obtained for surrogate earthquake catalogs (obtained by combining 24 plots similar to the one in panel a) together). Overplotted by the red dashed curve is a Gaussian distribution with the mean value equal to 0 and the standard deviation equal to 1 , demonstrating that the values of normalized probabilistic intensity follow this distribution.

their standard deviations to obtain "normalized probabilistic intensities" [20]. The resulting normalized probabilistic intensities allow us to directly evaluate whether the intensity in a given bin is less/more intense than average, and how statistically significant this effect is in terms of the number of standard deviations. This normalization benefits from the fact that according to the central limit theorem, the probabilistic intensities have a nearly normal distribution. Although an infinite number of cumulative probabilities need to be averaged for the probabilistic intensities to follow exactly a normal distribution, it turns out that averaging of only three cumulative probabilities results in the distribution of probabilistic intensities very close to normal (with an obvious limitation of extremely low/large probabilistic intensities, which cannot occur in our statistics, albeit the normal distribution predicts nonzero probability density for them).

The validity of the performed analysis was verified using surrogate earthquake catalogs. We started with the real earthquake catalog, and we constructed in total 24 surrogate catalogs by shifting the times of individual earthquakes by $\pm 30, \pm 60$, $\pm 90, \pm 120, \pm 150, \pm 180, \pm 210, \pm 240, \pm 270, \pm 300, \pm 330$, and \pm 360 days. Taking into account the considered time scales of possible effects related to the seismic activity, the applied time shifts are large enough to ensure that the data measured close to these surrogate virtual earthquakes should not be in any way affected by the seismic activity. Consequently, the main aim is to verify that the values of normalized probabilistic intensity indeed follow a normal distribution, and to demonstrate the noise level in resulting distance-time plots. The results obtained for these surrogate earthquake catalogs and the NWC transmitter are shown in Figure 6. Figure 6a shows normalized probabilistic intensities obtained for a single surrogate earthquake catalog. The results obtained for other surrogate earthquake catalogs are qualitatively the same. The normalized probabilistic intensities are color coded as a function of the time relative to the times of the (virtual) main shocks (abscissa) and distance of the NWC transmitter-DEMETER great circle paths from the respective earthquake epicenters (ordinate). As expected, one can see a significant scatter of the normalized probabilistic intensity values, both positive and negative, as corresponds to the Gaussian distribution they should follow. Considering that a surrogate earthquake catalog was used to obtain this plot, the observed variations are in no way related to the seismic activity, and they are strictly a statistical fluctuation. Figure $6 \mathrm{~b}$ 
(a)

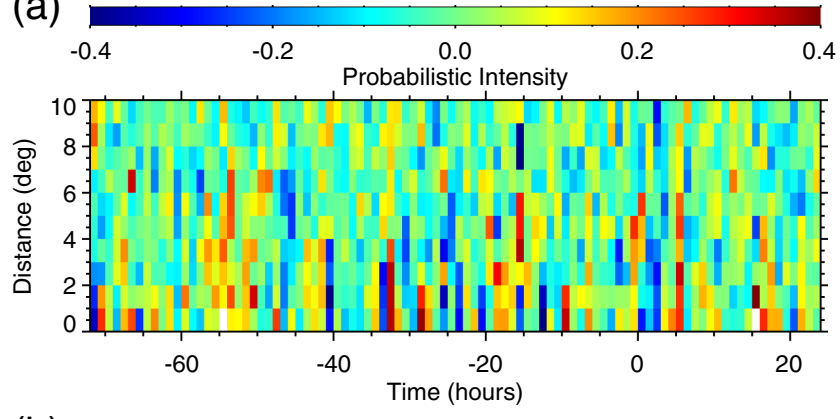

(b)

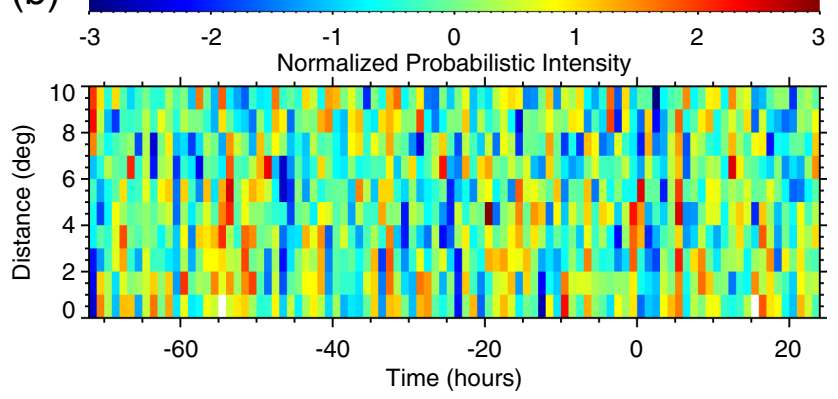

(c)

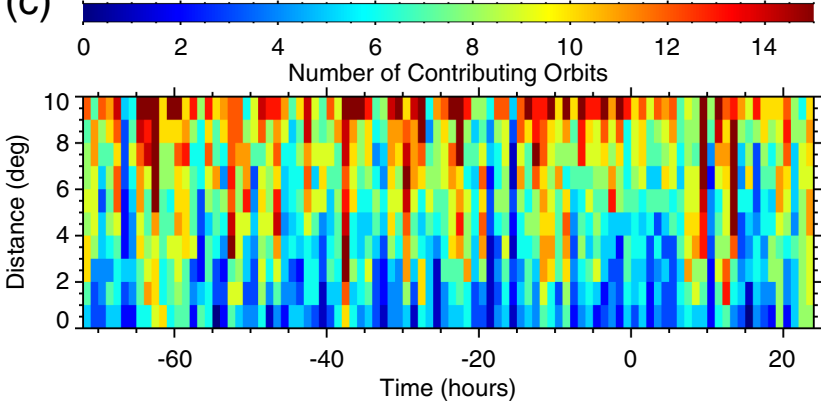

Fig. 7. Color coded as a function of the time relative to the times of the main shocks (abscissa) and distance of the NWC transmitter-DEMETER great circle paths from earthquake epicenters (ordinate). (a) Probabilistic intensity. (b) Normalized probabilistic intensity. (c) Number of DEMETER half-orbits contributing to the statistics.

demonstrates that the values of normalized probabilistic intensity indeed follow a Gaussian distribution with expected parameters. It was obtained by combining the normalized probabilistic intensities corresponding to all 24 surrogate earthquake catalogs together and plotting the resulting distribution. Overplotted by the red dashed line is a normal distribution with the mean value equal to 0 and the standard deviation equal to 1 , demonstrating that the values of normalized probabilistic intensities obtained for surrogate earthquake catalogs indeed follow this expected distribution.

\section{Results}

The resulting plot of the probabilistic intensity obtained for the NWC transmitter signal intensity and the real earthquake catalog is shown in Figure 7a. The probabilistic intensity in this plot is color coded according to the color scale at the top 
as a function of the time to/from an earthquake (abscissa) and distance between the earthquake epicenter and the NWC-DEMETER great circle path (ordinate). Although the values may in principle range between -0.5 and 0.5 , the real obtained range is somewhat lower due to the averaging. It can be seen that the behavior is rather noisy, in particular at lower distances where the amount of data in individual bins is quite low. However, focusing on effects lasting longer than one time bin, two apparently most significant effects identified in the figure are: (i) a decrease of the wave intensity at low distances (within about 4 degrees) shortly (0-3 hours) after the times of the main shocks, and (ii) an increase of the wave intensity at low distances observed about 50-57 hours before the times of the main shocks, followed by a subsequent intensity decrease. Figure $7 \mathrm{~b}$ uses the same format as Figure $7 \mathrm{a}$ to represent the dependence of the normalized probabilistic intensity. It can be seen that although the normalized probabilistic intensity values exhibit significant noise-like fluctuations, in agreement with their expected statistical behavior, the aforementioned intensity variations identified in the probabilistic intensity at low distances remain arguably the most pronounced effects. Figure 7c then shows the number of DEMETER half-orbits contributing to individual distance-time bins. This number is typically rather low, in particular at low distances. Unfortunately, this is a principal limitation given by the DEMETER orbit and transmitter/earthquake locations, and it can be in no way overcome by the performed analysis. Altogether, 715 seismic events contributed to the statistics of the NWC transmitter signal intensity variations.

Considering that the observed intensity variations possibly related to seismic activity occur - as it might be expected - at low distances, we further limit the analysis only to the data obtained at distances less than $4^{\circ}$ and use the same processing method but without an explicit dependence on the distance. This allows us to depict the results using line dependencies, as it is done in Figure 8. The black line in Figure 8a shows the time dependence of the probabilistic intensity, while the black line in Figure $8 \mathrm{~b}$ shows the time dependence of the normalized probabilistic intensity. It can be seen that the normalized probabilistic intensity is generally within about \pm 2 standard deviations. Nevertheless, both the intensity decrease just after the main shock times and the intensity variations (first increase, then decrease) about 50 hours before the main shock times are quite unique in the sense that they are not observed in single isolated bins, but they rather span over several consecutive time intervals. This correspondingly increases the statistical significance of the effects. In order to express this quantitatively, one might use 3-hours long time bins in place of the 1-hour long time bins, which would result in the dependencies plotted by the red lines in Figures $8 \mathrm{a}$ and $8 \mathrm{~b}$. The two aforementioned effects are thus the most striking features detected. Their statistical significance is, however, only about 2 standard deviations, i.e., there is about $5 \%$ chance that any of them occurred randomly. The number of contributing DEMETER half-orbits depicted in Figure 8c shows that they are formed by some 25 events.

Exactly the same data processing as for the NWC transmitter was performed also for the JJI transmitter signal. The obtained results are depicted in Figure 9. Although the position of the JJI transmitter is favorable for the intended analysis, its output power is lower than the output power of the NWC transmitter. Additionally, the frequency of the JJI transmitter is higher than the Nyquist frequency of the DEMETER VLF measurements. The transmitter signal is thus detected only due to the frequency aliasing, and its intensity is further decreased by the low-pass frequency filters applied onboard. All in all, the number of data relevant for the analysis of a possible seismic influence on the JJI transmitter signal intensity is lower than in the case of the NWC transmitter, as can be seen when comparing Figures $9 \mathrm{c}-8 \mathrm{c}$. In fact, the number of relevant events is so low that the color representation using the format of Figure 7 is nearly impossible, as it contains many distance-time bins with (nearly) 
(a)

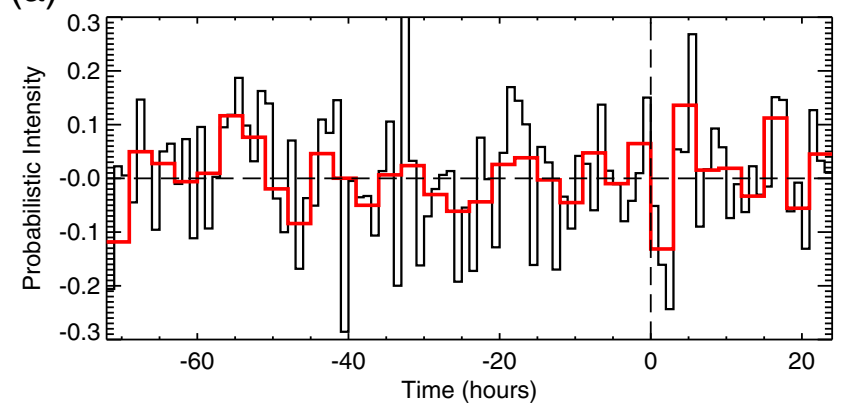

(b)

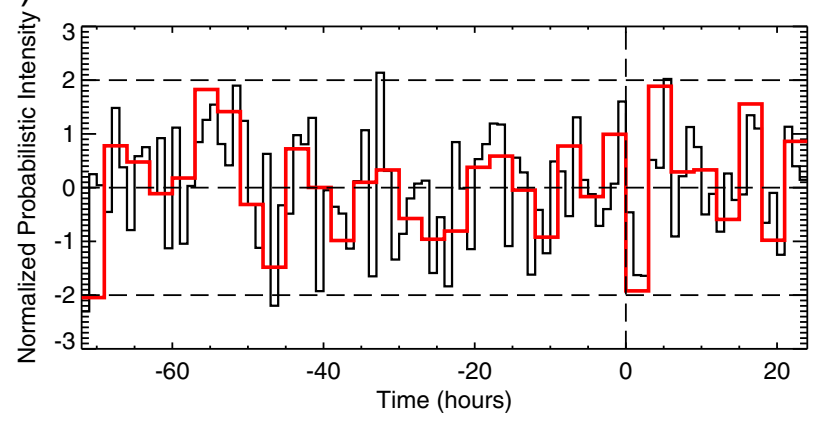

(c)

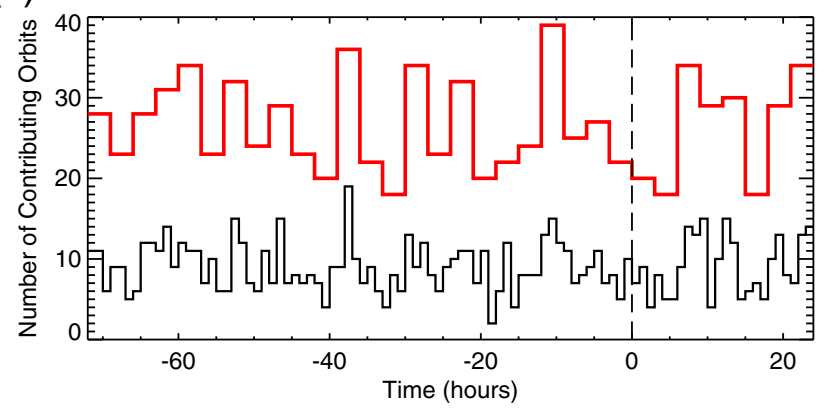

Fig. 8. Line representation of the results from Figure 7 at distances between the NWC transmitter-DEMETER great circle paths and earthquake epicenters lower than 4 degrees: (a) Probabilistic intensity. (b) Normalized probabilistic intensity. (c) Number of DEMETER half-orbits contributing to the statistics. The time resolution of the dependencies plotted in black is 1 hour, the time resolution of the dependencies plotted in red is 3 hours.

no data. Consequently, only the line representation is used for the JJI transmitter results. The total number of earthquakes contributing to the statistics in the case of the JJI transmitter is 316 . Figures $9 \mathrm{a}$ and $9 \mathrm{~b}$ show that - although the number of events is low - the decrease of the wave intensity just after the times of the main shocks observed previously for the NWC transmitter is well detectable also in the case of the JJI transmitter. This is a significant supporting piece of evidence that this effect is indeed real. The intensity increase well before the main shock times, followed by a subsequent decrease, which was observed for the NWC transmitter is not apparent in the JJI transmitter results. However, a decrease of the JJI transmitter signal intensity is observed about 48-51 hours before the main shock times, roughly 
(a)

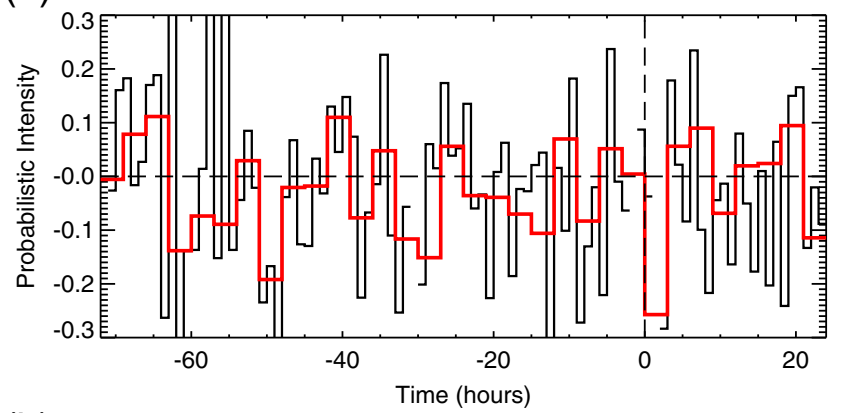

(b)

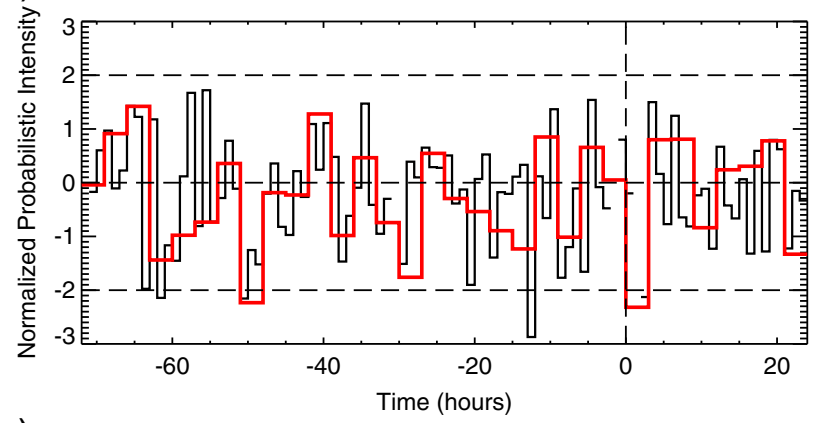

(c)

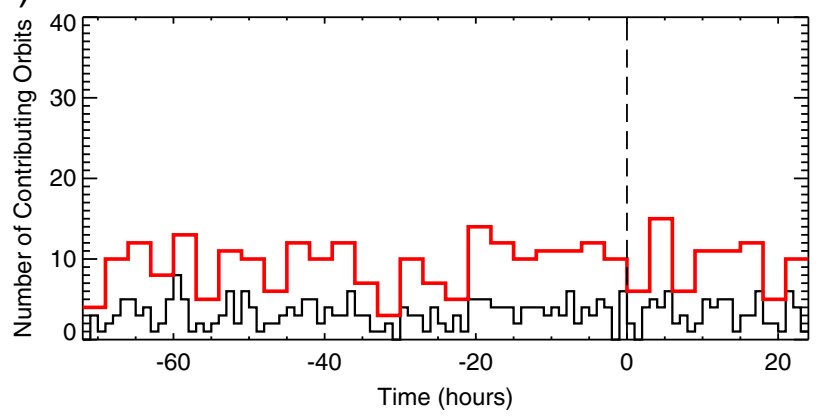

Fig. 9. Same as Figure 8, but for the results related to the JJI transmitter signal intensity. Note that the occasional gaps in the black curve in the are due to no data in these particular bins.

corresponding to the times when the decrease in the NWC transmitter signal intensity is observed.

\section{Discussion}

The observed decreases of the transmitter signal intensity might be consistent with the effect reported formerly [19] using a different data processing method. A comparable decrease of natural signal intensities at frequencies of about $1.7 \mathrm{kHz}$, i.e., close to the nighttime cut-off frequency of the Earth-ionosphere waveguide, was also reported [20-23]. These results suggest that around the times of strong shallow earthquakes, there might be significant changes at the bottom of the ionosphere, which, in turn, affect the wave propagation in the Earth-ionosphere waveguide [31]. An activation 
of highly mobile electronic charge carriers in rocks that are subjected to increasing levels of stress causes positive surface potential and field-ionization of air molecules [6]. The observed wave intensity decrease could be then due to the absorption of VLF electromagnetic waves by the $\mathrm{O}_{2}^{+}$molecules in the rising plume of ionized air (out of the air molecules, $\mathrm{O}_{2}$ has the lowest ionization potential, and, moreover, it has a dipole moment and, hence, can couple to the electromagnetic field). However, a considerable difference between our former and recent results is that while the formerly reported intensity decrease at frequencies of about $1.7 \mathrm{kHz}$ occurred shortly (0-4 hours) before the times of the main shocks, the VLF transmitter signal intensity decrease we reveal occurs after the main shock times. The origin of this difference remains unclear at the moment. However, we note that the analyzed effect is quite different. The formerly reported intensity decrease before main shock times was observed for natural electromagnetic emissions in a wide frequency range around the epicenter location, possibly related to an excess of ionization above the epicenter areas. On the other hand, the intensity decrease after main shock times we report on in the present study is observed for monochromatic artificial VLF transmitter signals at higher frequencies propagating in the Earth-ionosphere waveguide above seismic zones.

The ionospheric variations might be possibly related to a complicated chain of processes termed litosphere-atmosphere-ionosphere coupling, which has been theoretically analyzed in several recent papers $[4,5,7]$. We note, however, that the number of relevant events entering the performed statistics is rather small, resulting in not entirely convincing statistical significance of the observed effects. We further note that the intensity decrease observed just after the main shock times both in the NWC and JJI transmitter signal data may be possibly explained directly by seismically generated acoustic-gravity waves influencing the bottom of the ionosphere, and thus, in turn, the wave propagation in the Earth-ionosphere waveguide.

The variations of the transmitter signal intensity observed in 1-hour resolution plots possibly related to the earthquake occurrence are rather weak, and, given the limited number of relevant events, statistically significant only at less than 2 standard deviations level. Furthermore, comparably strong intensity variations were found also for other times and distances. Nevertheless, these other intensity variations seem unlikely to be related to the seismic activity, either because they only appear at large distances, or because the intensity in the neighboring time intervals is significantly different. On the other hand, the reported intensity variations possibly related to the seismic activity seem to be limited to low distances of the earthquake epicenters from the transmitter-DEMETER great circle path, and they span over several consecutive time bins. Taking this into account, and considering 3-hours resolution plots, the statistical significance of the observed phenomena is about 2 standard deviations. This means that there is about $5 \%$ chance that such intensity variations might have occurred randomly. Additionally, the intensity decrease after the main shocks was revealed independently for two different VLF transmitters, which is an important additional piece of evidence that the effect is indeed real and related to the earthquake occurrence.

Unfortunately, the available data set is rather limited, and it does not allow us to obtain more firm conclusions. While DEMETER acquired a huge amount of data during its mission, our analysis is limited only to the data from a relatively small area close to the transmitters. Given the geometry of the situation, the amount of the usable data is on the order of 10 half-orbits in each of the bins. At the lowest distances between the earthquake epicenters and the transmitter-DEMETER great circle paths, which are arguably most important for our analysis, the number of contributing half-orbits is even lower. Additionally, the amount of data further decreases for earthquakes with larger magnitudes, which makes it very hard to investigate 
whether the observed effect would be stronger for stronger earthquakes or not. For the same reason, no attempt to divide the earthquakes according to their type and/or hypocenter location below landmass/shoreline/ocean has been done.

The observed decreases of the transmitter signal intensity appear to be consistent with formerly reported transmitter signal variations related to the seismic activity, which also typically revealed some sort of an intensity decrease (e.g., [19,38,41-44, $46,47])$. At least for the intensity decrease observed after the main shock times, one may likely readily adopt the explanation based on the influence of atmospheric gravity waves propagating from an earthquake region [19]. On the other hand, the increase of the wave intensity well before the main shock times observed in the NWC transmitter data might be due to a change of the lower ionosphere resulting in a lower attenuation of signals propagating in the Earth-ionosphere waveguide. Such a change could eventually be a result of the litosphere-atmosphere-ionosphere coupling processes (see, e.g., $[4,5,7]$ and references therein). However, given that the intensity increase is not reproduced in the JJI transmitter results, a possibility that it might be only a statistical fluctuation cannot be excluded.

The identified variations of the VLF transmitter signal intensity are statistically significant only at about 2 standard deviations level, i.e., there is about $5 \%$ change that they might have occurred randomly. Moreover, they were obtained by combining together the data measured during the entire DEMETER mission. Considering individual events, the intensity variation may be clearly rather different than the average behavior obtained in the present study. Although our results thus indicate that there might be a relation between the seismic activity and VLF wave intensity measured by satellites in the upper ionosphere, their application to individual events or even to a short-term earthquake prediction does not appear to be feasible at the moment. We believe that the use of the DEMETER data was in this sense pushed nearly to its boundaries. In order to provide a definite conclusion on the existence of seismic related effects on the wave intensity in the upper ionosphere, it would strongly help to have another satellite similar to DEMETER (the more similar to DEMETER, the better - and the more satellites, the better). This would allow us to perform the same analyses using independent data set(s). Only the effects reproduced by these independent analyses should be then considered as real. In fact, one such satellite, China Seismo-Electromagnetic Satellite (CSES), was recently (February 2018) launched [58], and we are looking forward to its data set and results.

\section{Conclusions}

We have analyzed a possible influence of the seismic activity on the intensity of VLF transmitter signals measured by the low-orbiting spacecraft. Due to the transmitter signal intensity issues, only the data measured during the nighttime were included in the analysis. Moreover, following former statistical results, we focused exclusively on earthquakes with magnitudes larger than or equal to 5.0 and depths of hypocenters shallower than $40 \mathrm{~km}$. We have used primarily the signal of the NWC transmitter, as it is the most powerful transmitter advantageously located close to seismically active regions. We applied a two-step data processing which allows us to account for intensity variations related to non-seismic factors, and to evaluate the statistical significance of the observed effects. The obtained statistical results suggest that there might be slight (about 2 standard deviations) intensity variations related to the earthquake occurrence observed at the times when the great circle path between the NWC transmitter and the DEMETER spacecraft is within about 4 degrees from the earthquake epicenter. Specifically, an intensity decrease shortly (0-3 hours) after the times of the main shocks significant at about 2 standard deviations level was 
observed and independently reconfirmed using the JJI transmitter data, although the number of relevant events was very low. Additionally, some NWC transmitter signal intensity variations were observed also before the times of the main shocks. These were, however, only partially reproduced by the JJI transmitter results. The relatively low number of events included in the analysis, along with rather weak magnitudes of the observed effects, are insufficient to unambiguously demonstrate the statistical significance of the obtained results. The observed intensity decrease just after the main shock times is consistent with acoustic-gravity waves propagating from the earthquake region and influencing the bottom of the ionosphere. The transmitter signal intensity variations observed before the main shock times might be a manifestation of pre-seismic litosphere-atmosphere-ionosphere coupling processes. However, they still remain to be confirmed and eventually fully explained.

DEMETER was operated by CNES and the corresponding staff are deeply acknowledged. We also thank J. J. Berthelier, who was the principal investigator of the electric field instrument. The data used in the study are available at the Centre de Données de la Physique des Plasmas (http://cdpp2.cnes.fr/cdpp). The earthquake data used in the analysis can be downloaded from http://www.ldeo.columbia.edu/ gcmt/projects/CMT/catalog/jan76_ dec13.ndk.

Publisher's Note The EPJ Publishers remain neutral with regard to jurisdictional claims in published maps and institutional affiliations.

\section{References}

1. O.A. Molchanov, M. Hayakawa, J. Geophys. Res. 103, 17489 (1998)

2. M. Hayakawa, O.A. Molchanov, Adv. Space Res. 26, 1273 (2000)

3. P. Lognonné, J. Artru, R. Garcia, F. Crespon, V. Ducic, E. Jeansou, G. Occhipinti, J. Helbert, G. Moreaux, P.-E. Godet, Planet. Space Sci. 54, 528 (2006)

4. S.A. Pulinets, K.A. Boyarchuk, Ionospheric Precursors of Earthquakes (Springer Verlag, Berlin, Heidelberg, 2005)

5. R.G. Harrison, K.L. Aplin, M.J. Rycroft, J. Atmos. Solar-Terr. Phys. 72, 376 (2010)

6. F. Freund, I.G. Kulahci, G. Cyr, J. Ling, M. Winnick, J. Tregloan-Reed, M.M. Freund, J. Atmos. Solar-Terr. Phys. 71, 1824 (2009)

7. F. Freund, J. Asian Earth Sci. 41, 383 (2011)

8. S.A. Pulinets, D.O. Ouzounov, A.V. Karelin, D.V. Davidenko, Geomag. Aeron. 55, 521 (2015)

9. M. Parrot, in Electromagnetic Noise Due to Earthquakes, Handbook of Atmospheric Electrodynamics, edited by H. Volland (CRC Press, Boca Raton, Fla. 1995), p. 95-116

10. M. Li, M. Parrot, J. Geophys. Res. Space Phys. 118, 3731 (2013)

11. V.I. Larkina, V.V. Migulin, O.A. Molchanov, I.P. Kharkov, A.S. Inchin, V.B. Schvetcova, Phys. Earth Planet. Int. 57, 100 (1989)

12. J. Tate, W. Daily, Phys. Earth Planet. Int. 57, 1 (1989)

13. O.N. Serebryakova, S.V. Bilichenko, V.M. Chmyrev, M. Parrot, L. Rauch, F. Lefeuvre, O.A. Pokhotelov, Geophys. Res. Lett. 19, 91 (1992)

14. O.A. Molchanov, O.A. Mazhaeva, A.N. Goliavin, M. Hayakawa, Ann. Geophys. 11, 431 (1993)

15. M. Parrot, J. Geophys. Res. 99, 23339 (1994)

16. M. Parrot, in Atmospheric and Ionospheric Electromagnetic Phenomena Associated with Earthquakes, Statistical Studies with Satellite Observations of Seismogenic Effects, edited by M. Hayakawa (Terra Scientific Publishing, Tokyo, 1999), pp. 685-695

17. M. Parrot, M.M. Mogilevsky, Phys. Earth Planet. Int. 57, 86 (1989)

18. M. Parrot, J. Achache, J.J. Berthelier, E. Blanc, A. Deschamps, F. Lefeuvre, M. Menvielle, J.L. Plantet, P. Tarits, J.P. Villain, Phys. Earth Planet. Int. 77, 65 (1993) 
19. O. Molchanov, A. Rozhnoi, M. Solovieva, O. Akentieva, J.J. Berthelier, M. Parrot, F. Lefeuvre, P.F. Biagi, L. Castellana, M. Hayakawa, Nat. Haz. Earth Syst. Sci. 6, 745 (2006)

20. F. Němec, O. Santolík, M. Parrot, J.J. Berthelier, Geophys. Res. Lett. 35, L05109 (2008)

21. F. Němec, O. Santolík, M. Parrot, J. Geophys. Res. 114, A04303 (2009)

22. D. Píša, F. Němec, M. Parrot, O. Santolík, Ann. Geophys. 55, 157 (2012)

23. D. Píša, F. Němec, O. Santolík, M. Parrot, M. Rycroft, J. Geophys. Res. 118, 5286 (2013)

24. T.R. Henderson, V.S. Sonwalkar, R.A. Helliwell, U.S. Inan, A.C. Fraser-Smith, J. Geophys. Res. 98, 9503 (1993)

25. C.J. Rodger, N.R. Thomson, R.L. Dowden, J. Geophys. Res. 101, 13369

26. M.A. Clilverd, C.J. Rodger, N.R. Thomson, J. Geophys. Res. 104, 28171 (1999)

27. J.N. Thomas, J.J. Love, M.J.S. Johnston, Phys. Earth Planet. Int. 173, 207 (2009)

28. J.N. Thomas, J.J. Love, K. Yumoto, Geophys. Res. Lett. 36, L16301 (2009)

29. Y. Hobara, M. Parrot, J. Atmos. Solar-Terr. Phys. 67, 677 (2005)

30. C.L. Kuo, J.D. Huba, G. Joyce, L.C. Lee, J. Geophys. Res. 116, A10317 (2011)

31. K.G. Budden, The Wave-Guide Mode Theory of Wave Propagation (LOGOS PRESS, London, 1961)

32. M. Hayakawa, Y. Kasahara, T. Nakamura, F. Muto, T. Horie, S. Maekawa, Y. Hobara, A.A. Rozhnoi, M. Solovieva, O.A. Molchanov, J. Geophys. Res. 115, A09305 (2010)

33. Y. He, D. Yang, J. Qian, M. Parrot, Nat. Haz. Earth Syst. Sci. 11, 2173 (2011)

34. M. Parrot, Earth. Sci. 24, 513 (2011)

35. M. Li, M. Parrot, Nat. Haz. Earth Syst. Sci. 12, 2957 (2012)

36. M. Parrot, Ann. Geophys. 55, 149 (2012)

37. R. Yan, M. Parrot, J.-L. Pinçon, J. Geophys. Res. Space Phys. 122, 12421 (2017)

38. M.Y. Boudjada, K. Schwingenschuh, R. Döller, A. Rohznoi, M. Parrot, P.F. Biagi, P.H.M. Galopeau, M. Solovieva, O. Molchanov, H. K. Biernat, G. Stangl, H. Lammer, I. Moldovan, W. Voller, M. Ampferer, Nat. Haz. Earth Syst. Sci. 10, 1487 (2010)

39. A. Rozhnoi, O. Molchanov, M. Solovieva, V. Gladyshev, O. Akentieva, J.J. Berthelier, M. Parrot, F. Lefeuvre, M. Hayakawa, L. Castellana, P.F. Biagi, Nat. Haz. Earth Syst. Sci. 7, 617 (2007)

40. A. Rozhnoi, M. Solovieva, M. Parrot, M. Hayakawa, P.F. Biagi, K. Schwingenschuh, Ann. Geophys. 55, 187 (2012)

41. A. Rozhnoi, M. Solovieva, M. Parrot, M. Hayakawa, P.F. Biagi, K. Schwingenschuh, V. Fedun, Phys. Chem. Earth Parts A/B/C 85-86, 141 (2015)

42. P.F. Biagi, L. Castellana, T. Maggipinto, D. Loiacono, V. Augelli, L. Schiavulli, A. Ermini, V. Capozzi, M.S. Solovieva, A.A. Rozhnoi, O.A. Molchanov, M. Hayakawa, Nat. Haz. Earth Syst. Sci. 8, 1041 (2008)

43. P.F. Biagi, L. Castellana, T. Maggipinto, G. Maggipinto, A. Minafra, A. Ermini, O. Molchanov, A. Rozhnoi, M. Solovieva, M. Hayakawa, Phys. Chem. Earth Parts A/B/C 34, 456 (2009)

44. E. Slominska, J. Blecki, M. Parrot, J. Slominski, Phys. Chem. Earth Parts A/B/C 34, 464 (2009)

45. M.S. Solovieva, A.A. Rozhnoi, O.A. Molchanov, Geomag. Aeron. 49, 532 (2009)

46. M. Hayakawa, Y. Kasahara, T. Nakamura, Y. Hobara, A. Rozhnoi, M. Solovieva, O.A. Molchanov, J. Atmos. Solar-Terr. Phys. 72, 982 (2010)

47. M.Y. Boudjada, P.F. Biagi, E. Al-Haddad, P.H.M. Galopeau, B. Besser, D. Wolbang, G. Prattes, H. Eichelberger, G. Stangl, M. Parrot, K. Schwingenschuh, Phys. Chem. Earth Parts A/B/C 102, 70 (2017)

48. A. Rozhnoi, M. Solovieva, O. Molchanov, P.F. Biagi, M. Hayakawa, K. Schwingenschuh, M. Boudjada, M. Parrot, Nat. Haz. Earth Syst. Sci. 10, 529 (2010)

49. J.J. Berthelier, M. Godefroy, F. Leblanc, M. Malingre, M. Menvielle, D. Lagoutte, J.Y. Brochot, F. Colin, F. Elie, C. Legendre, P. Zamora, D. Benoist, Y. Chapuis, J. Artru, R. Pfaff, Planet. Space Sci. 54, 456 (2006)

50. U.S. Inan, M. Gołkowski, D.L. Carpenter, N. Reddell, R.C. Moore, T.F. Bell, E. Paschal, P. Kossey, E. Kennedy, S.Z. Meth, Geophys. Res. Lett. 31, L24805 (2004) 
51. M. Gołkowski, U.S. Inan, A.R. Gibby, M.B. Cohen, J. Geophys. Res. 113, A10201 (2008)

52. M.B. Cohen, U.S. Inan, J. Geophys. Res. 117, A08310 (2012)

53. F. Němec, O. Santolík, M. Parrot, J. Bortnik, J. Geophys. Res. 113, A08317 (2008)

54. X. Tao, J. Bortnik, M. Friedrich, J. Geophys. Res. 115, A07303 (2010)

55. M.B. Cohen, N.G. Lehtinen, U.S. Inan, Geophys. Res. Lett. 39, L24101 (2012)

56. K.L. Graf, N.G. Lehtinen, M. Spasojevic, M.B. Cohen, R.A. Marshall, U.S. Inan, J. Geophys. Res. Space Phys. 118, 2708 (2013)

57. M.L. Hutchins, A.R. Jacobson, R.H. Holzworth, J.B. Brundell, J. Geophys. Res. Space Phys. 118, 5808 (2013)

58. X. Shen, X. Zhang, S. Yuan, L. Wang, J. Cao, J. Huang, X. Zhu, P. Piergiorgio, J. Dai, Science Chin. Technol. Sci. 61, 634 (2018) 